\title{
Proton spin relaxation in methyl substituted aromatic radical ions. A formula for the $\mathrm{CH}_{3}$ proton NMR linewidth
}

\author{
J. W. M. de Boer, M. R. Arick, and E. de Boer \\ Department of Physical Chemistry, University of Nijmegen, Toernooiveld, Nijmegen. The Netherlands
}

(Received 24 August 1972)

\begin{abstract}
The proton relaxation has been studied in methyl substituted aromatic radical ions in solution. The linewidth parameter $T_{2}$ of the protons in such radicals is governed by two intramolecular interactions, namely, the Fermi contact interaction and the anisotropic dipolar interaction. For the methyl protons the interactions are modulated in time by both internal rotation and tumbling of the whole molecule in solution. Equations have been derived to account for the effect of these motions on the $\mathrm{CH}_{3}$ proton linewidth. The resulting formulas are used to analyze the NMR proton linewidths for the anion of 3, 3'dimethylbiphenyl.
\end{abstract}

\section{INTRODUCTION}

Internal rotation in molecules has been the subject of many investigations during the last decades. Especially rotation of methyl groups was studied, its effects can be observed in ir, Raman, 1 and microwave spectroscopy, ${ }^{2}$ in the thermodynamic properties ${ }^{3}$ of molecules, in slow neutron scattering experiments ${ }^{4}$ and in ESR spectra of methyl containing radicals. ${ }^{5,6}$ In the ESR spectra the $\mathrm{CH}_{3}$ rotation manifested itself clearly by a change of the hyperfine pattern due to the $\mathrm{CH}_{9}$ group on decreasing the temperature.

Since internal motion is able to modulate magnetic interactions, methyl rotation may act as a relaxation mechanism for the $\mathrm{CH}_{9}$ proton spins and accordingly may influence the line shape of nuclear magnetic resonance signals. This has given rise to a number of NMR studies on diamagnetic compounds. Our interest in the subject arose from a recent article by La Mar and Van Hecke ${ }^{7}$ which reports an NMR study on hindered methyl rotation in paramagnetic $\mathrm{Cr}$ (II) complexes containing a $\mathrm{CH}_{9}$ group in the ligands. They suggest that the relaxing effect of internal rotation can be accounted for by a simple modification of the linewidth formulas valid for protons that are rigidly fixed to the molecule; only the electron correlation time $\tau_{e}$ should be replaced by $\tau_{e}^{\prime}=\left(\tau_{e}^{-1}+\tau_{m}^{-1}\right)^{-1}$, where $\tau_{m}$ is the correlation time for the internal rotation. If this is true, one would expect to find motional narrowing of the proton resonance lines, when $\tau_{m}<\tau_{e}$. As La Mar and Van Hecke pointed out, this motional narrowing has not been observed, but they suggested that it would manifest itself in aromatic free radicals, for which both $\tau_{e}$ and the coupling constants are larger than those in the complexes investigated by them.

These statements inspired us to start investigations on methyl substituted aromatic radical an-
Ions. In order to explain our results, it proved necessary to derive equations which describe in a proper way the influence of methyl rotation on the linewidth. The derived equations show that the intuitive formulas of La Mar and Van Hecke are not correct. In Sec. II a detailed account of the derivation of the linewidth formulas is given; in Sec. IV the results of our experiments on the anion of 3, 3'-dimethylbiphenyl (dmb) are discussed.

\section{THEORETICAL}

In general the relaxation of proton spins depends on time dependent interactions of both inter- and intramolecular origin. For a proton in a paramagnetic molecule the intramolecular interactions between the unpaired electron spin and the proton spins are by far the most important. Taking these interactions into account, an expression for the linewidth parameter $T_{2}$ valid for protons fixed to the molecule was derived. ${ }^{8-10}$ For protons undergoing internal rotation no satisfactory formula for $T_{2}^{-1}$ has been given. In Sec. II. A the formulas are summarized which hold for the ring protons; in Sec. II. B formulas are derived which are valid for the protons of the $\mathrm{CH}_{3}$ group.

\section{A. Ring Protons}

For protons fixed to the rigid molecular frame of a paramagnetic molecule, the linewidth parameter $T_{2}$ is governed by two contributions

$$
T_{2}^{1}=\left(T_{2}^{-1}\right)_{F C}+\left(T_{2}^{-1}\right)_{D}
$$

the first term stands for the Fermi contact interaction and the second term for the anisotropic electron dipole-nuclear dipole interaction. For an aromatic radical ion in solution, undergoing rapid Brownian motion, the following expressions have been derived ${ }^{8-10}$ :

$$
\begin{aligned}
& \left(T_{2}^{-1}\right)_{\mathrm{FC}}=\frac{1}{4}(A)^{2} \tau_{e}\left[1+1 /\left(1+\omega_{e}^{2} \tau_{e}^{2}\right)\right] \\
& \left(T_{2}^{-1}\right)_{D}=\frac{1}{20}(B)^{2} \tau_{d}\left[7+13 /\left(1+\omega_{e}^{2} \tau_{d}^{2}\right)\right]
\end{aligned}
$$


where $A$ is the hyperfine splitting constant (hfsc) of the proton (in radians per seconds) and $B$ (in radians per seconds) $=\gamma_{e} \gamma_{N} \hbar / r^{3}$ in a point dipole model, where $r$ is the distance between the dipoles. The electron angular frequency is denoted by $\omega_{e}$, the electron spin relaxation time by $\tau_{e}$ and the dipolar correlation time by $\tau_{d}$, where

$$
\tau_{d}^{-1}=\tau_{e}^{-1}+\tau_{r}^{-1}
$$

and $\tau_{r}$ is the correlation time for molecular reorientation.

If we are dealing with a delocalized electron, it was shown, ${ }^{11}$ that Eq. (2b) still holds, if $B$ is defined by

$$
B^{2}=\frac{1}{6} \operatorname{Tr} \mathrm{T}^{2}
$$

where $T$ stands for the anisotropic dipolar tensor; its elements can be written as

$$
T_{\alpha \beta}=\left\langle\psi\left|\left[\left(r^{2} \delta_{\alpha \beta}-3 r_{\alpha} r_{\beta}\right) / r^{5}\right]\right| \psi\right\rangle \gamma_{e} \gamma_{N} \hbar
$$

$\psi$ is the odd electron orbital wave function; $\alpha, \beta$ $=1,2,3$, and $r_{1}, r_{2}, r_{3}$ are the $x, y$, and $z$ components of the proton-electron radius vector.

\section{B. $\mathrm{CH}_{3}$ Protons}

\section{Formulation of the Problem}

The protons of a $\mathrm{CH}_{3}$ group possess an extra degree of freedom with respect to the ring protons, because rotation is possible around the threefold axis. Since this internal rotation modulates the interactions with the unpaired electron, it may function as an extra relaxation mechanism.

Freed $^{5}$ has given a detailed study of the influence of quantum effects of methyl group rotations on linewidths of hyperfine lines in ESR spectra. Here it is necessary to consider explicitly the phase coherency between the protons of the methyl group, since the electron spin relaxation is governed by the interaction with the three proton nuclei of the rotating $\mathrm{CH}_{3}$ group. In our case, however, the relaxation of the proton spin is governed by the interaction with the single spin of the unpaired electron; mutual interactions between the proton spins of the $\mathrm{CH}_{3}$ group can be discarded (see Appendix). The density matrix formalism described by Abragam can be used to derive a rather general expression for the linewidth of a proton interacting with one unpaired electron. ${ }^{10}$ For our purposes it is sufficient to consider the case where the electron spin $\mathbf{S}$ relaxes fast compared to the proton spin I; the spin $S$ can be taken as part of the lattice. It can be shown that the time dependent interaction Hamiltonian $\mathfrak{F}_{1}(t)$ including the Fermi contact and the anisotropic dipolar interaction can generally be written as

$$
\mathfrak{K}_{1}(t)=\sum_{q} F^{(q)}(t) I_{q} \quad q=-1,0,+1
$$

where $F^{(\alpha)}$ and $I_{q}$ describe the lattice and the nuclear spin variables, respectively $\left(I_{ \pm}=I_{x} \pm i I_{y}\right.$ and $\left.I_{0}=I_{R}\right)$. The behavior of the spin $I$ is properly described by Bloch equations and for $T_{2}^{-1}$ it is found ${ }^{10}$ :

$$
T_{2}^{-1}=J_{1}\left(\omega_{I}\right)+\frac{1}{2} J_{0}(0) \text {. }
$$

The $J_{q}(\omega)$ are Fourier transforms of the correlation functions $g_{q}(\tau)$ :

$$
\begin{aligned}
& g_{q}(\tau)=\left\langle F^{(a)}(t) F^{(-q)}(t+\tau)\right\rangle \\
& J_{q}(\omega)=\int_{-\infty}^{\infty} g_{q}(\tau) e^{-t \omega \tau} d \tau .
\end{aligned}
$$

The brackets in Eq. (8) indicate an ensemble average over all molecules in the solution. The relevant interaction Hamiltonian reads as

$$
\mathfrak{K}_{1}(t)=\mathbf{S}(t) \cdot[A(t) 1+\mathbf{T}(t)] \cdot \mathbf{I} .
$$

The first term describes the Fermi contact interaction ( 1 is the unit dyadic) and the second term the anisotropic dipolar interaction.

Because the proton spin is coupled to the external magnetic field by the Zeeman interaction, all quantities in Eq. (10) are given in the laboratory frame. The time dependence of $\mathbf{S}$ is governed by the electron spin relaxation, that of $A$ by internal rotation and that of $T$ by both internal and overall rotations (tumbling of the whole molecule in the solution). Writing the Hamiltonian of Eq. (10) in the form of Eq. (6) yields

$$
\begin{aligned}
F^{(0)}(t)=\left[A(t)+F_{0}(t)\right] S_{z}(t) & \\
& +F_{1}(t) S_{+}(t)+F_{1}^{*}(t) S_{-}(t), \\
F^{(1)}(t)=F_{1}(t) S_{g}(t) & +F_{2}(t) S_{+}(t) \\
& +\left[\frac{1}{2} A(t)-\frac{1}{4} F_{0}(t)\right] S_{-}(t), \\
F^{(-1)}(t)=F_{1}^{*}(t) S_{z}(t) & +F_{2}^{*}(t) S_{-}(t) \\
& +\left[\frac{1}{2} A(t)-\frac{1}{4} F_{0}(t)\right] S_{+}(t),
\end{aligned}
$$

where the $F_{q}$ are functions of nuclear coordinates only, defined by

$$
\begin{aligned}
& F_{0}=T_{33}, \\
& F_{1}=\frac{1}{2}\left[T_{13}-i T_{23}\right], \\
& F_{2}=\frac{1}{4}\left[T_{11}-T_{22}-2 i T_{12}\right] .
\end{aligned}
$$

\section{Correlation Functions}

We are now left with the calculation of the correlation functions $g_{q}(\tau)$ obtained by substitution of Eq. (11) into Eq. (8), and their Fourier transforms. This can be done assuming that the averages over electron spin motion and nuclear rotational motion can be separated. Using the fact that correlation functions of the form $\left\langle S_{\lambda}(t)\right.$ $\left.\times S_{\mu}(t+\tau)\right\rangle(\lambda, \mu=z,+,-)$ vanish unless $S_{\mu}$ is the Hermitian conjugate of $S_{\lambda}$, this yields: 


$$
\begin{aligned}
& g_{0}(\tau)=\left\langle\left[F_{0}(t)+A(t)\right]\left[F_{0}(t+\tau)+A(t+\tau)\right]\right\rangle\left\langle S_{\varepsilon}(t) S_{\varepsilon}(t+\tau)\right\rangle \\
&+\left\langle F_{1}(t) F_{1}^{*}(t+\tau)\right\rangle\left\langle S_{+}(t) S_{-}(t+\tau)\right\rangle+\left\langle F_{1}^{*}(t) F_{1}(t+\tau)\right\rangle\left\langle S_{-}(t) S_{+}(t+\tau)\right\rangle
\end{aligned}
$$

and

$$
\begin{aligned}
g_{1}(\tau)=g_{-1}(\tau)=\left\langle F_{1}(t) F_{1}^{*}(t+\tau)\right\rangle\left\langle S_{g}(t) S_{g}(t+\tau)\right\rangle & +\left\langle F_{2}(t) F_{2}^{*}(t+\tau)\right\rangle\left\langle S_{+}(t) S_{-}(t+\tau)\right\rangle \\
& +\frac{1}{4}\left\langle\left[\frac{1}{2} F_{0}(t)-A(t)\right]\left[\frac{1}{2} F_{0}(t+\tau)-A(t+\tau)\right]\right\rangle\left\langle S_{-}(t) S_{+}(t+\tau)\right\rangle .
\end{aligned}
$$

The electron spin correlation functions are easy to handle when $\mathrm{S}$ can be described by Bloch equations with relaxation times $\tau_{1}$ and $\tau_{2}$. One obtains ${ }^{10}$

$$
\begin{aligned}
\left\langle S_{z}(t) S_{z}(t+\tau)\right\rangle & =[S(S+1) / 3] \exp \left\{-|\tau| / \tau_{1}\right\} \\
\left\langle S_{+}(t) S_{-}(t+\tau)\right\rangle & =\left\langle S_{-}(t) S_{+}(t+\tau\rangle^{*}\right. \\
& =\frac{2}{3} S(S+1) \exp \left\{-i \omega_{\theta} \tau-\left(|\tau| / \tau_{2}\right)\right\} .
\end{aligned}
$$

Furthermore, since $A(t)$ depends on internal motion only, and $F_{0}=T_{g a}$ is an element of a traceless tensor, terms involving $\left\langle A(t) F_{0}(t+\tau)\right\rangle$ and $\left\langle F_{0}(t) A(t+\tau)\right\rangle$ vanish. Thus Eqs. (13) and (14) become

$$
\begin{aligned}
g_{0}(\tau)=\frac{1}{3} S(S+1)\left\{\left[\left\langle F_{0}(t) F_{0}(t+\tau)\right\rangle+\langle A(t) A(t+\tau)\rangle\right] \exp \left(-|\tau| / \tau_{1}\right)\right. \\
\left.+2\left[e^{-i \omega_{e} \tau}\left\langle F_{1}(t) F_{1}^{*}(t+\tau)\right\rangle+e^{i \omega_{e} \tau}\left\langle F_{1}^{*}(t) F_{1}(t+\tau)\right\rangle\right] \exp \left(-|\tau| / \tau_{2}\right)\right\}
\end{aligned}
$$

and

$$
\begin{aligned}
& g_{1}(\tau)=g_{-1}(\tau)=\frac{1}{3} S(S+1)\left\{\left\langle F_{1}(t) F_{1}^{*}(t+\tau)\right\rangle \exp \left(-|\tau| / \tau_{1}\right)+2\left[e^{i \omega_{e} \tau\left(\frac{1}{16}\left\langle F_{0}(t) F_{0}(t+\tau)\right\rangle+\frac{1}{4}\langle A(t) A(t+\tau)\rangle\right)}\right.\right. \\
&\left.\left.+e^{-i \omega_{e} \tau}\left\langle F_{2}(t) F_{2}^{*}(t+\tau)\right\rangle\right] \exp \left(-|\tau| / \tau_{2}\right)\right\} .
\end{aligned}
$$

From these equations one sees that the Fermi contact and the dipolar contribution to $T_{2}^{1}$ are independent. They may be calculated separately and simply added afterwards.

\section{Autocorrelation Functions}

The problem is now reduced to the calculation of the autocorrelation functions for $A(t)$ and for $F_{q}(t)$. Let us first consider the $F_{q}$. In Eq. (10) all quantities are expressed in coordinates with respect to a laboratory fixed reference frame (RF), so are the functions $F_{q}$. Transformation of the $F_{q}$ to a molecule fixed $R F$ with the aid of the Wigner rotation matrices yields expressions, in which the time dependence due to the tumbling motion of the molecules is contained completely in the transformation matrices; the time dependence of the transformed parameters is only due to internal motions. In evaluating the autocorrelation functions serious difficulties are encountered since the rotational diffusion equation has to be solved analytically. It is more convenient to transform each coordinate in Eq. (5) separately using three RF's as was introduced by Perrin. ${ }^{12}$

The reference frames are defined as follows: $S$ is the laboratory fixed RF; $S^{\circ}$ is a $R F$ with a fixed orientation with respect to $S$ and its axes define the initial orientation of a molecule at time $t$; $S^{\prime}$ is a molecule fixed RF giving the orientation of the molecule at times $t+\tau(\tau \geqslant 0) . \quad S^{\circ}$ and $S^{\prime}$ coincide at time $t(\tau=0)$. Further let $\Omega=(\varphi, \vartheta, \psi)$ be the set of Euler angles ${ }^{13}$ relating $S^{\circ}$ to $S$ and $\Omega_{0}=\left(\varphi_{0}, \vartheta_{0}, \psi_{0}\right)$ such a set relating $S^{\prime}$ to $S^{\circ}$. Ac- cordingly, $\Omega$ is a function of $t$ and $\Omega_{0}$ of $\tau$ only. $\mathbf{r}, \mathbf{r}^{\circ}, \mathbf{r}^{\prime}$ are the electron-proton radius vectors in the frames $S, S^{0}$, and $S^{\prime}$, respectively. Then we have

$$
\begin{aligned}
& r=a(\Omega) r^{0} \\
& r^{\circ}=c\left(\Omega_{0}\right) r^{\prime}
\end{aligned}
$$

where a and c are rotation matrices; their elements are given in Refs. 12 and 14 in terms of the Euler angles.

Now we can write

$$
\begin{aligned}
& \mathrm{r}(t)=\mathrm{a}(t) \mathrm{r}^{\circ}(t)=\mathrm{a}(t) \mathrm{r}^{\prime}(t) \\
& \mathrm{r}(t+\tau)=\mathrm{a}(t) \mathrm{r}^{\circ}(t+\tau)=\mathrm{a}(t) \mathrm{c}(\tau) \mathrm{r}^{\prime}(t+\tau)
\end{aligned}
$$

For a given time $t, a(t)$ gives the initial orientation of a molecule, $\mathrm{c}(\tau)$ contains all information on overall rotation and $\mathbf{r}^{\prime}(t+\tau)$ involves only internal motions [note that $\mathrm{c}(0)$ is the unit dyadic $\operatorname{sir}_{2}$ ?e $S^{\prime}$ and $S^{\circ}$ coincide for $\tau=0$ ].

Then from Eq. (5) and Eq. (12) we find (introducing the symbol $D$ for the dipolar tensor in the molecule fixed RF $S^{\prime}$ ):

$$
\begin{aligned}
& T_{i j}(t+\tau)=\sum_{k, l, m, n} a_{i k}(t) a_{j l}(t) \\
& \times c_{k m}(\tau) c_{l n}(\tau) D_{m n}(t+\tau)
\end{aligned}
$$

and after some mathematical manipulations one finds 


$$
\begin{aligned}
& \left\langle F_{q}(t) F_{q}^{*}(t+\tau)\right\rangle=\sum_{k^{\alpha}, l^{d}, k, l, m, n}\left\langle\alpha_{k^{\prime} l^{\prime}, k l}^{(q)}(t) c_{k m}(\tau) c_{l n}(\tau)\right. \\
& \text { where } \quad 9=0,1,2 \\
& \left.\times D_{k^{*} l^{\bullet}}(t) D_{m n}(t+\tau)\right\rangle \\
& \alpha_{k^{*} l l^{*} k l}^{(0)}=a_{3 k^{\prime}}, a_{3 l}, a_{3 k} a_{3 l} \\
& \alpha_{k^{\prime} l^{*} k l}^{(1)}=A_{k^{\prime}}^{*} a_{3 l} \cdot A_{k} a_{3 l} \\
& \alpha_{k^{\prime} l^{\prime} k 1}^{(2)}=A_{k^{\prime}}^{*}, A_{1}^{*}, A_{k} A_{l}
\end{aligned}
$$

with

$$
A_{k}=\left(a_{1 k}+i a_{2 k}\right) / 2
$$

It can be shown that the rotational Hamiltonian for a system undergoing both overall and internal rotation can be written without interaction terms between the two motions by a proper choice of angular momenta. ${ }^{2}$ Thus the two motions can be considered as independent and the averages over them can be separated. In addition the averaging over all initial orientations of the molecules involves only the $\alpha_{k^{\prime} l^{\prime} k l}^{(q)}$; hence we can rewrite Eq. (21) to

$$
\begin{aligned}
& \left\langle F_{q}(t) F_{q}^{*}(t+\tau)\right\rangle=\sum_{k^{1}, l^{0}, k_{1}, l_{1} m_{1}, n}\left\langle\alpha_{k^{\prime} l^{\prime}, k l}^{(q)}(t)\right\rangle \\
& \times\left\langle c_{k m}(\tau) c_{l n}(\tau\rangle\left\langle D_{k^{\prime} l^{\prime}}(t) D_{m n}(t+\tau)\right\rangle .\right.
\end{aligned}
$$

The averages on the right-hand side of Eq. (23) were calculated as follows:

(a) The first average can be obtained by simple integration assuming a random distribution of orientations at time $t$ :

$$
\left\langle\alpha_{k^{\prime} l l_{k l}(q)}(t)\right\rangle=\int d \Omega\left(1 / 8 \pi^{2}\right) \alpha_{k}^{(q)}{ }_{k l}(\Omega) .
$$

The symmetry properties of the $a_{k t}{ }^{14}$ can be used to reduce the number of integrals to be calculated explicitly.

(b) The average over overall rotation involves the conditional probability $P\left(\Omega_{0}, \tau\right)$ of finding a molecule rotated after a time $\tau$ over the angles $\Omega_{0}$ from its orientation $\Omega$ at time $t$. The great advantage of Perrins method is that it does not require any knowledge of $P\left(\Omega_{0}, \tau\right)$ except that it satisfies the rotational diffusion equation for Brownian motion

$$
\partial P\left(\Omega_{0}, \tau\right) / \partial \tau=\Lambda P\left(\Omega_{0}, \tau\right),
$$

where $\Lambda$ is a second order differential operator in $\varphi_{0}, \vartheta_{0}$, and $\psi_{0}$ (see Eq. (17) of Ref. 14). Now for any function $f$ regarding the molecular orientation one finds ${ }^{12}$

$$
d\langle f\rangle / d \tau=\langle\Lambda f\rangle \text {. }
$$

Perrin ${ }^{12}$ has shown that the nonvanishing rotation averages contain products of elements of the matrix c that are eigenfunctions of $\Lambda$, e.g.,

$$
\Lambda c_{i i}(\tau)^{2}=\left(1 / \tau_{r}\right) c_{i i}(\tau)^{2} .
$$

Using the initial condition $c_{i j}(0)=\delta_{i j}$ and the orthogonality of c one finds with Eq. (25):

$$
\begin{aligned}
& \left\langle c_{i i}(\tau)^{2}\right\rangle=\frac{1}{3}\left[1+2 \exp \left(-|\tau| / \tau_{r}\right)\right] \\
& \left\langle c_{i j}(\tau)^{2}\right\rangle=\frac{1}{3}\left[1-\exp \left(-|\tau| / \tau_{r}\right)\right], \quad i \neq j \\
& \left\langle c_{i t}(\tau) c_{j \jmath}(\tau)\right\rangle+\left\langle c_{i j}(\tau) c_{j i}(\tau)\right\rangle=\exp \left(-|\tau| / \tau_{r}\right), \\
& \quad i \neq j .
\end{aligned}
$$

All other averages vanish by symmetry.

Substitution of the results of (a) and (b) into Eq. (23) gives

$$
\begin{aligned}
\left\langle F_{q}(t) F_{q}^{*}(t+\tau)\right\rangle=k_{q} & \exp \left(-|\tau| / \tau_{r}\right) \\
& \times \sum_{i, j}\left\langle D_{i j}(t) D_{i j}(t+\tau)\right\rangle
\end{aligned}
$$

with $k_{0}=2 / 15, k_{1}=1 / 20=k_{2}$.

(c) The average $\left\langle D_{i j}(t) D_{i j}(t+\tau)\right\rangle$ can be written as

$$
\begin{aligned}
\left\langle D_{i j}(t) D_{i j}(t+\tau)\right\rangle= & \int_{0}^{2 \pi} d \varphi_{0} P\left(\varphi_{0}\right) D_{i j}\left(\varphi_{0}\right) \\
& \times \int_{0}^{2 \pi} d \varphi P\left(\varphi_{0}, \varphi, \tau\right) D_{i j}(\varphi),
\end{aligned}
$$

where $P\left(\varphi_{0}, \varphi, \tau\right)$ is the conditional probability of finding the methyl proton at angle $\varphi$ at time $t+\tau$, when it was at $\varphi_{0}$ at time $t$ (see Fig. 1). In order to perform the integration analytical expressions are needed for $D_{i j}(\varphi)$ and $P\left(\varphi_{0}, \varphi, \tau\right)$.

In a rotation diffusion model for $\mathrm{CH}_{3}$ rotation, $P(\varphi, 0, \tau)$ may be obtained from the diffusion equation

$$
\partial P / \partial \tau=R_{m}\left(\partial^{2} P / \partial \varphi^{2}\right)
$$

with $R_{m}$ as the diffusion constant for internal rotation. A solution of Eq. (29) is the Gaussian distribution ${ }^{15}$ :

$$
P\left(\varphi_{0}, \varphi, \tau\right)=\frac{1}{2}\left(\tau_{m} / \pi \tau\right)^{1 / 2} \exp \left\{-\varphi^{\prime 2} \tau_{m} / 4 \tau\right\},
$$

where $\varphi^{\prime}=\varphi-\varphi_{0}$ and $\tau_{m}=1 / R_{m}$.

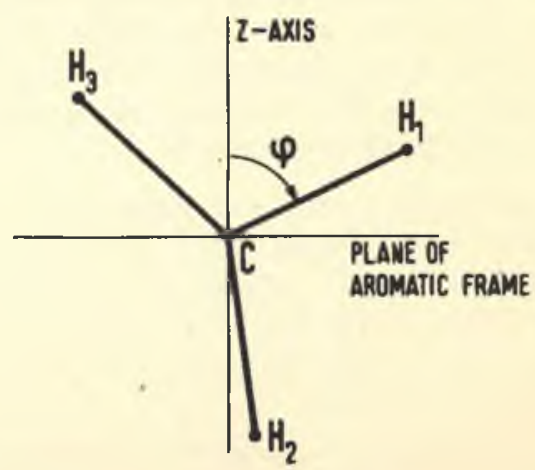

FIG. 1. Definition of the methyl group orientation angle $\varphi$. 
Analytical expressions for the dipolar tensor elements $D_{i j}(\varphi)$ are not so easily found, mainly because of the $\varphi$ dependence in $r^{\prime 5}$ [see Eq. (5)]. Since the averaging over electronic coordinates was done numerically, it was convenient to calculate $D_{i j}$ for a number of angles $\varphi(0 \leqslant \varphi \leqslant 2 \pi)$. After that $D_{i j}(\varphi)$ were expanded in a Fourier series:

$$
D_{i j}(\varphi)=\sum_{k=-N}^{N} D_{i j}^{(k)} e^{i k \varphi} .
$$

The number of terms to be taken into account (i.e., the value of $N$ ) depends on the specific case; in practice rapid convergency is reached. Since the dipolar tensor is real, one has $D_{i j}^{(k)}=D_{i j}^{(-k) *}$. The result of this Fourier expansion is, that we can write

$$
\left\langle D_{i j}(t) D_{i j}(t+\tau)\right\rangle=\sum_{k, l=-N}^{N} D_{i j}^{(k)} D_{i j}^{(l)}\left\langle e^{i k \varphi(t)} e^{i 1 \varphi(t+r)}\right\rangle
$$

and the average on the right-hand side of this equation may be evaluated by integration, using Eq. (30) and assuming that there is a random distribution of initial orientations, thus $P\left(\varphi_{0}\right)=1 / 2 \pi$. One then obtains

$$
\begin{aligned}
\langle\exp \{i k \varphi(t)\} \exp \{i l \varphi(t+\tau)\}\rangle & =\delta_{k,-l} \\
& \times \exp \left\{-k^{2}\left(|\tau| / \tau_{m}\right)\right\}
\end{aligned}
$$

so that finally Eq. (27) becomes

$$
\left\langle F_{q}(t) F_{q}^{*}(t+\tau)\right\rangle=6 k_{q} \sum_{k=-N}^{N} B_{k}^{2} \exp \left(-|\tau| / \tau_{r k}\right),
$$

where

and

$$
B_{k}^{2}=\frac{1}{6} \sum_{l, j}\left|D_{l j}^{(k)}\right|^{2}=\frac{1}{6} \operatorname{Tr}\left\{\left|D^{(k)}\right|^{2}\right\}
$$

$$
\tau_{r h}^{-1}=\tau_{r}^{-1}+k^{2} \tau_{m}^{-1}
$$

The autocorrelation function for $A(t)$ is now readily obtained. Expanding $A(\varphi)$ in a Fourier series

$$
A(\varphi)=\sum_{k=-N^{*}}^{N^{0}} \alpha_{k} e^{i k \varphi}
$$

and using Eq. (32) one gets

$$
\begin{aligned}
\langle A(t) A(t+\tau)\rangle= & \sum_{k=-N^{\circ}}^{N^{\prime}}\left|\alpha_{k}\right|^{2} \exp \left(-k^{2}|\tau| / \tau_{m}\right) . \\
& \text { 4. Linewidth Equation }
\end{aligned}
$$

If we now substitute the results, that is, Eqs. (33), (35) into Eqs. (16), (17), perform the Fourier transformation of Eq. (9), substitute the found $J_{q}(\omega)$ into Eq. (7) and separate the contributions due to the Fermi contact and the dipolar interaction, we finally get

$$
\left(T_{2}^{-1}\right)_{\mathrm{FC}}=\sum_{k=-N^{*}}^{N^{*}} \frac{1}{4}\left|\alpha_{h}\right|^{2}\left[\tau_{k 1}+\tau_{k 2} /\left[1+\left(\omega_{e}-\omega_{1}\right)^{2} \tau_{k 2}^{2}\right]\right]
$$

anci

$$
\begin{aligned}
\left(T_{2}^{-1}\right)_{D}= & \sum_{k=-N}^{N} \frac{1}{20} B_{k}^{2}\left[\tau_{k d 1}\left\{4+\frac{3}{1+\omega_{I}^{2} \tau_{k d 1}^{2}}\right\}\right. \\
& +\tau_{k d 2}\left\{\frac{6}{1+\omega_{e}^{2} \tau_{k d 2}^{2}}+\frac{1}{1+\left(\omega_{e}-\omega_{I}\right)^{2} \tau_{k d 2}{ }^{2}}\right. \\
& \left.\left.+\frac{6}{1+\left(\omega_{e}+\omega_{I}\right)^{2} \tau_{k d 2}{ }^{2}}\right\}\right]
\end{aligned}
$$

where

$$
\tau_{k 1}^{-1}=\tau_{1}^{-1}+k^{2} \tau_{m}^{-1},\left(\tau_{k d 1}\right)^{-1}=\tau_{1}^{-1}+\tau_{r}^{-1}+k^{2} \tau_{m}^{-1}
$$

and similar expressions for $\tau_{k 2}$ and $\tau_{k d 2}$ replacing $\tau_{1}$ by $\tau_{2}$. The above equations can be reduced considerably using the conditions valid in our case: $\omega_{I} \ll \omega_{e}, \omega_{I}^{2} \tau_{k d 1}^{2} \ll 1$ and $\tau_{1}=\tau_{2} \equiv \tau_{e}$. Furthermore in a planar aromatic radical $A(\varphi)$ is fairly well described by ${ }^{5,16}$

$$
A(\varphi)=a+a \cos 2 \varphi .
$$

Thus $\alpha_{0}=a, \alpha_{-2}=\alpha_{2}=a / 2$ and all other $\alpha_{k}$ vanish. Thus

$$
\begin{aligned}
\left(T_{2}^{-1}\right)_{F C} & =\sum_{k} \frac{\left|\alpha_{k}\right|^{2}}{4} \tau_{k e}\left[1+\frac{1}{1+\omega_{e}^{2} \tau_{k e}^{2}}\right] \\
& =\frac{a^{2}}{4} \tau_{e}\left[1+\frac{1}{1+\omega_{e}^{2} \tau_{e}^{2}}\right]+\frac{a^{2}}{8} \tau_{2 e}\left[1+\frac{1}{1+\omega_{e}^{2} \tau_{2 e}^{2}}\right] \\
\times\left(T_{2}^{-1}\right)_{D} & =\sum_{k} \frac{B_{k}^{2}}{20} \tau_{k d}\left[7+\frac{13}{1+\omega_{e}^{2} \tau_{h d}^{2}}\right]
\end{aligned}
$$

with

$$
\begin{aligned}
& \tau_{k a}^{-1}=\tau_{a}^{-1}+k^{2} \tau_{m}^{-1} \\
& \tau_{k d}^{-1}=\tau_{a}^{-1}+k^{2} \tau_{m}^{-1} .
\end{aligned}
$$

From the definition of $\tau_{k d}, \tau_{k d}$ and Eq. (37) it is seen that the $\mathrm{CH}_{3}$ proton linewidth is predicted to vary with $\tau_{m}$ only in a certain range of $\tau_{m}$ values, viz for $\tau_{m} \approx \tau_{e}, \tau_{d}$ (vide infra, Fig. 7). In the limit of fast internal rotation $\left(\tau_{m} \ll \tau_{e}, \tau_{d}\right)$ all $\tau_{k e}$ and $\tau_{k d}$ vanish for $k \neq 0$. Hence Eq. (37) reduces then essentially to Eq. (2) involving only the mean values $\alpha_{0}$ and $B_{0}$ of the methyl proton hfsc and the dipolar tensor, respectively; very fast $\mathrm{CH}_{3}$ rotation provides no effective relaxation mechanism. In the other limit $\left(\tau_{m} \gg \tau_{e}, \tau_{d}\right)$ all $\tau_{k e}, \tau_{k d}$ tend to $\tau_{e}$, $\tau_{d}$, respectively, and the contributions to the linewidth become

$$
\begin{aligned}
& \left(T_{2}^{-1}\right)_{\mathrm{YC}}=(3 / 8) a^{2} \tau_{e}\left[1+1 /\left(1+\omega_{e}^{2} \tau_{e}^{2}\right)\right] \\
& \left(T_{2}^{-1}\right)_{D}=(1 / 20)\left(\sum B_{k}^{2}\right) \tau_{d}\left[7+13 /\left(1+\omega_{e}^{2} \tau_{d}^{2}\right)\right] .
\end{aligned}
$$

However, the theory given here only holds for fast internal rotation, that is $\mathrm{CH}_{3}$ rotation much faster than the maximum resonance frequency difference $\Delta \nu=2 a$ [Eq. (36); the extremes are found for $\varphi=0$ and $\varphi=\pi / 2]$. For a methyl hfsc of $0.5 \mathrm{Oe}$ this means $\tau_{m} \ll 10^{-6} \mathrm{sec}$. 


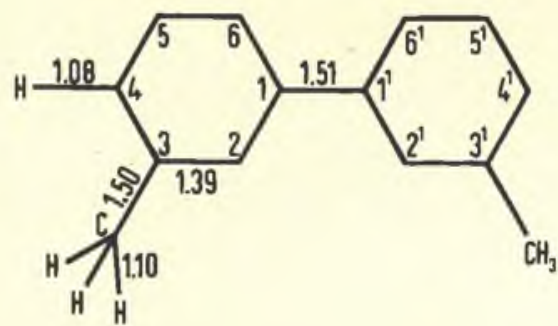

FIG. 2. 3, 3'-dimethylbiphenyl (dmb): numbering system. Bond lengths (in $\AA$ ) are indicated.

Finally, the above theory predicts no motional narrowing in the fast rotational limit. The $\mathrm{CH}_{3}$ proton linewidth is calculated to be always larger than or equal to the linewidth predicted from Eq. (2), in contradiction to the equations given by $\mathrm{La}$ Mar and Van Hecke, which predict always a smaller linewidth.

\section{EXPERIMENTAL}

The proton NMR measurements on the anion of $\mathrm{dmb}$ were performed on a Varian DP60 external lock (EL) spectrometer, operating at $60 \mathrm{MHz}$. The temperature of the samples was controlled by a V4540 unit and measured with a thermocouple. The samples were prepared by high vacuum standard techniques. ${ }^{17}$ The temperature range that could be studied was limited by the high freezing point of the solution (about $-10^{\circ} \mathrm{C}$ ) and by the fact that radical decomposition started at temperatures above $40^{\circ} \mathrm{C}$. The radical concentration in the NMR tube could be varied by distilling solvent out of or into this tube. The concentration was determined by measurement of the volume of the solution by means of a calibrated tube (with an accuracy of $0.1 \mathrm{ml}$, that is $2 \%-5 \%$ ).

The degree of reduction, the fraction of aromatic molecules that were reduced to radical anions, was determined by measuring the shift of the solvent signals with respect to their positions in the unreduced dmb solution. Reduction on the sodium mirror was continued until the solvent shift reached its maximum. To observe the solvent peaks the spectrometer was operated in the high resolution mode, whilst the detection of the proton resonances of the dmb anion was done in the wide line (WL) mode. The signals of the methyl and meta protons ( $3,3^{\prime}$ and $5,5^{\prime}$, see Fig. 2) were so narrow that modulation corrections ${ }^{18,19}$ on the linewidth were necessary. In spite of this disadvantage we wereforced to measure these peaks in the WL mode, because in the high resolution mode overlap between peaks and sidebands arising from frequency modulation made accurate measurements impossible. The modulation corrections were obtained from the figures given in Ref. 19. It ac- counted for the effects of both amplitude and frequency of the field modulation. The observed linewidths were also corrected for intermolecular broadening determined by the paramagnetic particles in the solution. This was accomplished by measuring the broadening of the solvent proton peaks in the reduced solutions with respect to those in the unreduced solutions.

The contact shifts were determined directly from the spectra using the position of the solvent peak as an internal reference.

In order to determine the shift and linewidth for the overlapping ortho proton peaks $\left(2,2^{\prime}\right.$ and $\left.6,6^{\prime}\right)$ a computer analysis was necessary. In the computer program NMRFIT the basic line shape is assumed to be derivative Lorentzian. The sum of the squares of the differences between the fitted spectrum and the measured one is calculated. The best set of parameters characterizing each line (shift, linewidth) is determined by minimizing the sum of the squared differences using a nonlinear minimization program MINUIT obtained from CERN (Geneve). It was verified that the results were insensitive to the values of the input parameters. In Fig. 3 is shown the result of one such fitting. The final calculated line, which is the sum of the two lines depicted, is within the noise of the measured spectrum.

The very broad para proton peak $\left(4,4^{\prime}\right)$ was difficult to measure and only a rough estimate of its position could be made.

$\mathrm{dmb}$ (Kogh-Light Ltd.) was purified once by vacuum distillation. The fraction between 114 and $118^{\circ} \mathrm{C}$ was collected $(4-5 \mathrm{~mm} \mathrm{Hg})$. The solvent used, 1, 2-dimethoxyethane, was dried over sodium,

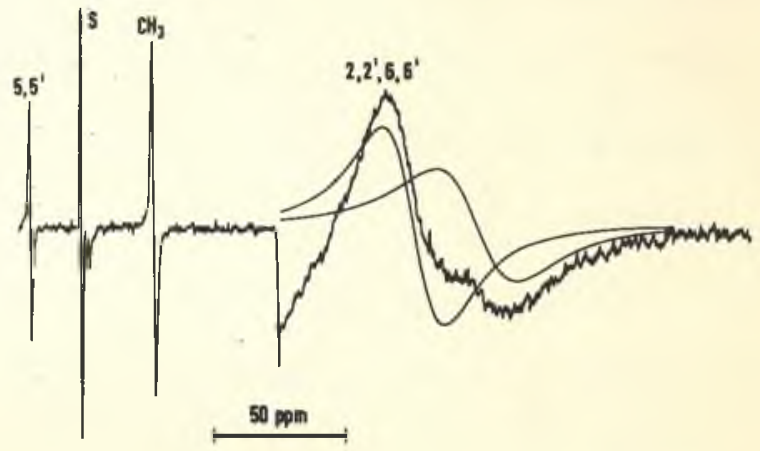

FIG. 3. WL NMR spectrum of the dmb anion in 1, 2dimethoxyethane. The numbers above the lines refer to the positions of the protons (Fig. 2). $S$ denotes the solvent peak. The ortho proton absorption line can be decomposed into two absorption lines (see text), which are also shown in the figure. The spectrum was obtained at $30^{\circ} \mathrm{C}$, and at a concentration of $1.6 \mathrm{M}$. 
TABLE I. Hyperfine splitting constants, spin densities, and dipolar interaction parameters for the anion of $\mathrm{dmb}$.

\begin{tabular}{lcccc}
\hline \hline Position & $\begin{array}{c}a_{H} \mathrm{NMR} \\
(\mathrm{Oe})\end{array}$ & $\begin{array}{c}\left|a_{H}\right| \mathrm{ESR} \\
(\mathrm{Oe})\end{array}$ & $\rho_{i}$ & $\begin{array}{c}B^{2} \\
(\mathrm{MHz} / \mathrm{sec})^{2}\end{array}$ \\
\hline $1,1^{\prime}$ & $\cdots$ & $\cdots$ & 0.127 & $\cdots$ \\
$2,2^{\prime}$ & -2.37 & 2.33 & 0.092 & 6.15 \\
$3,3^{\prime}\left(\mathrm{CH}_{3}\right)$ & -0.494 & 0.49 & -0.017 & 0.66 \\
$4,4^{\prime}$ & -5.25 & 5.15 & 0.203 & 27.3 \\
$5,5^{\prime}$ & +0.284 & 0.27 & -0.011 & 1.64 \\
$6,6^{\prime}$ & -2.78 & 2.82 & 0.107 & 7.44 \\
\hline \hline
\end{tabular}

vacuum distilled and stored in vacuo over a $\mathrm{Na}-\mathrm{K}$ alloy.

\section{RESULTS AND DISCUSSION}

\section{A. Hyperfine Splitting Constants, Spin Densities}

The measurement of the hfsc is required for the linewidth analysis for two reasons. Firstly, the Fermi contact contribution to the linewidth is determined by the coupling constants, secondly, calculation of the $T$ tensor and $B^{2}$ (that appear in the dipolar contribution) requires knowledge of the spin density distribution in the radical, which may be inferred in part from the hfsc.

Table I shows the hfsc of the protons in the dmb anion obtained from NMR contact shifts. ${ }^{20,21}$ The assignment of the coupling constants for the ortho protons was based on theoretical calculations that yield a higher spin density on carbon atoms $6,6^{\prime}$; thus the larger coupling constant was assigned to the $6,6^{\prime}$ protons and the smaller to the $2,2^{\prime}$ protons. The absolute values of the hfsc are close to those obtained by $\mathrm{ESR}^{22}$; complete agreement was not even expected, since our NMR experiments were performed on 1-2 $M$ solutions, while ESR measurements usually use concentrations of about $10^{-4} M^{23}$

The spin densities $\rho_{l}$ on the carbon atoms $2,4,5,6$ and the equivalent ones in the other ring are related to the hfsc $a_{i}$ of the protons on those positions by the well known McConnell relation ${ }^{24}$ :

$$
a_{i}=Q_{\alpha} \rho_{i}
$$

where $Q_{\alpha}$ is a proportionality constant with a value of -26 Oe for aromatic molecules. A similar equation was proposed by Fessenden and Schuler ${ }^{25}$ to relate the hfsc of a $\mathrm{CH}_{3}$ proton to the spin density on the carbon atom to which the methyl group is attached:

$$
a_{\beta}=Q_{\beta} \rho \text {. }
$$

Using this relation $\rho_{3}, \rho_{3}$, can be calculated $\left(Q_{\beta}\right.$ is set equal to $+29.3 \mathrm{Oe}^{25}$ ). The spin densities $\rho_{1}, \rho_{1}$, are found from the normalization of the total spin density.

\section{B. Calculation of T Tensors}

The tensor for the anisotropic dipolar interaction $T$ was calculated in a semiempirical way. It has only five independent elements, since it is traceless and symmetric [Eq. (5)]. In the spin density matrix formalism one can write ${ }^{26,27}$

$$
\mathrm{T}=\sum_{i, j} \rho_{i j} \mathrm{~T}_{i j},
$$

where the summation runs over an appropriate set $\left\{\varphi_{i}\right\}$ of atomic orbitals. For this the set of $2 p_{z}-\mathrm{AO}^{\prime} s$ of the aromatic carbon atoms was used (which in fact implies the neglect of the influence of the alkali counterion). Furthermore, the spin density matrix was assumed to be diagonal, that means neglect of overlap between functions of the set $\left\{\varphi_{i}\right\}$. Then Eq. (40) reduces to

$$
\mathbf{T}=\sum_{i} \rho_{i i} \mathbf{T}_{i i} .
$$

The tensor $\mathbf{T}_{i}$ is the tensor for the dipolar interaction between the proton under consideration and an electron spin localized in the $2 p_{z}$ orbital of carbon atom $i$. It was calculated with the formulas developed by McConnell and Strathde ${ }^{26}$ and Derbyshire, ${ }^{28}$ using an effective nuclear charge for the carbon atoms of 3.1358 in the Slater $2 p_{z}$ orbitals. ${ }^{29}$ The experimental spin densities $\rho_{i}$ of Table I were used as the diagonal density matrix elements $\rho_{i i}$. The geometry of $\mathrm{dmb}$ has not yet been determined, so it was assumed that the biphenyl skeleton is not disturbed by the introduction of the methyl groups and consists of two coplanar regular hexagons of carbon atoms with bond lengths ${ }^{30}$ as indicated in Fig. 2. The methyl protons were put on the three corners of a tetrahedron with the methyl carbon atom in the center; bond lengths, shown in Fig. 2 were estimated from a number of interatomic distances obtained for other aromatic methyl derivatives.

The $T$ tensor for a methyl proton in the dmb anion was calculated for a number of angles $\varphi(0 \leqslant \varphi \leqslant 2 \pi)$ and the resulting values were analyzed by a computer program that calculated the Fourier coefficients $D_{i j}^{(k)}$ of Eq. (31). While testing the program with known sums of sines and cosines we found that many points (intervals between the points smaller than, say, $0.5 \mathrm{deg}$ ) were required to get accurate results (deviations less than about $1 \%$ from the real coefficients). Calculating the $B_{k}^{2}$ values for $\mathrm{dmb}$ (in units of $10^{-12} \mathrm{rad}^{2} / \mathrm{sec}^{2}$ ), we found

$$
\begin{aligned}
& B_{0}^{2}=25.5, \\
& B_{1}^{2}=0.2, \\
& B_{2}^{2}=0.5, \\
& B_{k}^{2}<0.01 \text { for } k \geqslant 3 .
\end{aligned}
$$




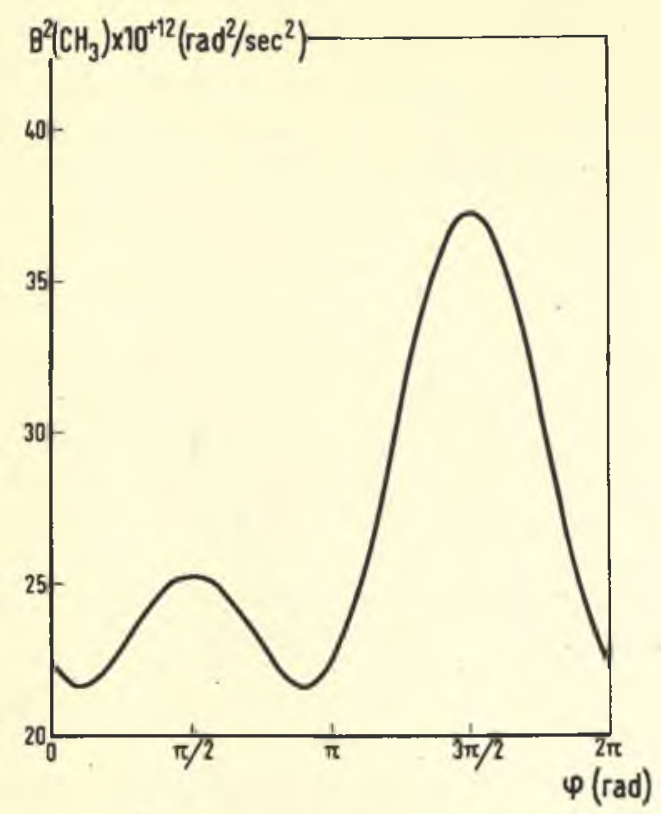

FIG. 4. $B^{2}$ as a function of $\varphi$ for a methyl proton in $\mathrm{dmb}$ anion.

From these results it is clear that for the anion of dmb the $\tau_{m}$ dependent part of the dipolar contribution to the linewidth (terms containing $B_{h}^{2}$, $k \neq 0)$ is small. The effect of methyl rotation on $T_{2}^{-1}$ is here mainly governed by the Fermi contact contribution.

In Fig. 4 the $\varphi$ dependence of $B^{2}(\varphi)=\frac{1}{6} \operatorname{Tr}\left[D^{2}(\varphi)\right]$ is shown. Two maxima are calculated: one when the proton is near the 2 position (the lower maximum; $\varphi=\pi / 2$ ), the other one (the higher maximum) when it is near the 4 position $(\varphi=3 \pi / 2)$, which carries the highest spin density.

\section{Ring Proton Linewidths}

The analysis of the ring proton linewidths with Eqs. (1)-(3) yields values for the correlation times $\tau_{\theta}, \tau_{d}$, and $\tau_{r}$. Substitution of Eq. (2) into Eq. (1) gives an equation with two unknowns $\tau_{e}$

TABLE II. Correlation times for the anion of dmb.

\begin{tabular}{cccc}
\hline$T$ & $C$ & $\tau_{e} \times 10^{11}$ & $\begin{array}{c}\tau_{d} \times 10^{11} \\
(\mathrm{sec})\end{array}$ \\
$\left({ }^{\circ} \mathrm{C}\right)$ & $(M)$ & $2.7 \pm 0.3^{\mathrm{a}}$ & $0.22 \pm 0.08^{\mathrm{a}}$ \\
\hline 30 & 1.25 & $2.5 \pm 0.3$ & $0.30 \pm 0.11$ \\
30 & 1.39 & $2.0 \pm 0.3$ & $0.17 \pm 0.05$ \\
30 & 1.67 & $1.4 \pm 0.2$ & $0.31 \pm 0.09$ \\
30 & 1.92 & $2.0 \pm 0.3$ & $0.58 \pm 0.20$ \\
27 & 1.67 & $2.1 \pm 0.3$ & $0.24 \pm 0.08$ \\
20 & 1.67 & $2.2 \pm 0.03$ & $0.30 \pm 0.11$ \\
10 & 1.67 & $2.9 \pm 0.4$ & $0.20 \pm 0.08$ \\
-2 & 1.67 & 2 & \\
\hline \hline
\end{tabular}

The error given is one standard deviation.

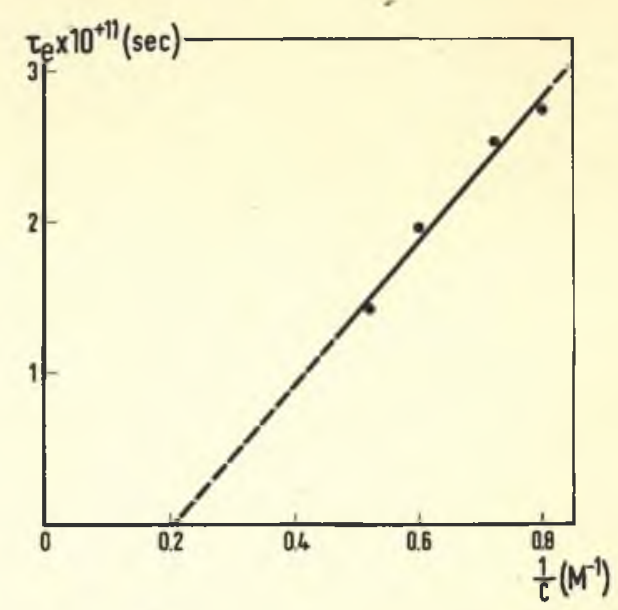

FIG. 5. Concentration dependence of the electron correlation time $\tau_{e}$ for the anion of $\mathrm{dmb}$ in 1,2-dimethoxyethane at $30^{\circ} \mathrm{C}$.

and $\tau_{d}$, that can be solved when the resonance signals of two inequivalent protons are measured. In order to calculate $\tau_{\theta}$ and $\tau_{d}$ we combined the results for the protons 2 and 5 and for 6 and 5 . It turned out that both combinations give almost the same values for the correlation times. Combining the results for the protons 2 and 6 leads to two nearly dependent equations, yielding nonreliable results. The values obtained for $\tau_{e}$ and $\tau_{d}$ for some concentrations and temperatures are given in Table II. The contributions of nonsecular parts of the time dependent Hamiltonian [the second terms in the brackets in Eq. (2)] were included since $\omega_{e}^{2} \tau_{e}^{2}, \omega_{e}^{2} \tau_{d}^{2} \approx 1\left(\omega_{e}=2.5 \times 10^{-11} \mathrm{sec}^{-1}\right.$ at the applied external magnetic field).

An analysis of the temperature dependence is not meaningful because of the small temperature range that could be studied and the rather high inaccuracies (see Table II). The expected linear relationship between $\tau_{e}$ and the inverse of the concentration $^{31}(1 / C)$ is affirmed by our experiments as Fig. 5 shows. In addition, the observed proportionality between the widths of the ortho proton lines and $1 / C$ (Fig. 6) points to the predominance of the Fermi contact conbribution to $T_{2}^{-1}$ as is expected for protons with large coupling constants. ${ }^{27,32}$ This is also the reason that the linewidth equations derived for the protons 2 and 6 are nearly dependent.

\section{Methyl Proton Linewidth}

The linewidth of the $\mathrm{CH}_{3}$ protons in the anion of dmb can be analyzed with the equations derived in Sec. II. At a given temperature and concentration $\tau_{e}$ and $\tau_{d}$ were determined from the linewidths of the ring protons. With the resulting values $T_{2}^{-1}$ for the $\mathrm{CH}_{3}$ protons can be predicted as a function 


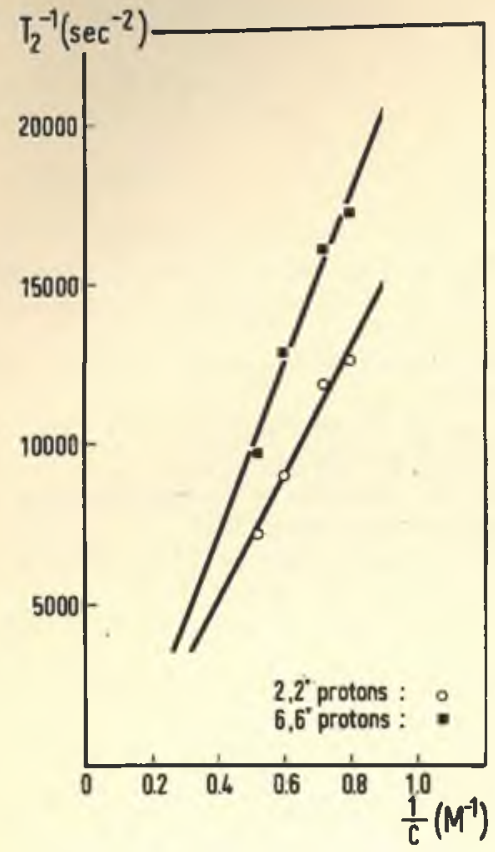

FIG. 6. Concentration dependence of the ortho-proton linewidth at $30^{\circ} \mathrm{C}$.

of $\tau_{m}$. Figure 7 shows this for a particular case $\left(30^{\circ} \mathrm{C} ; 1.9 \mathrm{M}\right)$. For $\tau_{m} \leq 10^{-12} \sec T_{2}^{1}$ is independent of the magnitude of $\tau_{m}$. This is the fast rotation limit, where the linewidth equations for the methyl protons [Eq. (37)] become identical with those for the ring protons [Eq. (2)]. The predicted linewidth increases rapidly when $\tau_{m}$ becomes larger than $10^{-11} \mathrm{sec}$ and it reaches the other limit for $\tau_{m} \simeq 10^{-8} \mathrm{sec}$. In Table III the results of the analysis are summarized. Given are the observed $T_{2}^{-1}$ and the predicted $T_{2}^{-1}$ in the fast $\left(\tau_{m} \ll \tau_{e}\right)$ and slow $\left(\tau_{m} \gg \tau_{e}\right)$ rotational limit. The errors in the predicted linewidths are about $15 \%$ to $20 \%$ mainly due to the uncertainty in the correlation times $\tau_{e}$ and $\tau_{d}$. At all temperatures and concentrations studied the observed $T_{2}^{-1}$ agrees within the experimental error

TABLE III. Experimental and theoretical linewidth parameters for the methyl protons in the anion of $\mathrm{dmb}$.

\begin{tabular}{ccccc}
\hline \hline & & $T_{2}^{-1}$ & \multicolumn{2}{c}{$\begin{array}{c}\text { Limits } T_{2}^{-1} \text { sec }^{-1} \\
\text { predicted }\end{array}$} \\
$\left({ }^{\circ} \mathrm{C}\right)$ & $C$ & $\begin{array}{c}\text { observed } \\
\left(\mathrm{sec}^{-1}\right)\end{array}$ & $\tau_{m}=0$ & $\tau_{m}=\infty$ \\
\hline 30 & 1.25 & 547 & 576 & 841 \\
30 & 1.39 & 570 & 552 & 798 \\
30 & 1.67 & 385 & 425 & 619 \\
30 & 1.92 & 341 & 350 & 495 \\
27 & 1.67 & 424 & 482 & 681 \\
20 & 1.67 & 447 & 462 & 669 \\
10 & 1.67 & 470 & 481 & 693 \\
-2 & 1.67 & 474 & 590 & 871 \\
\hline \hline
\end{tabular}

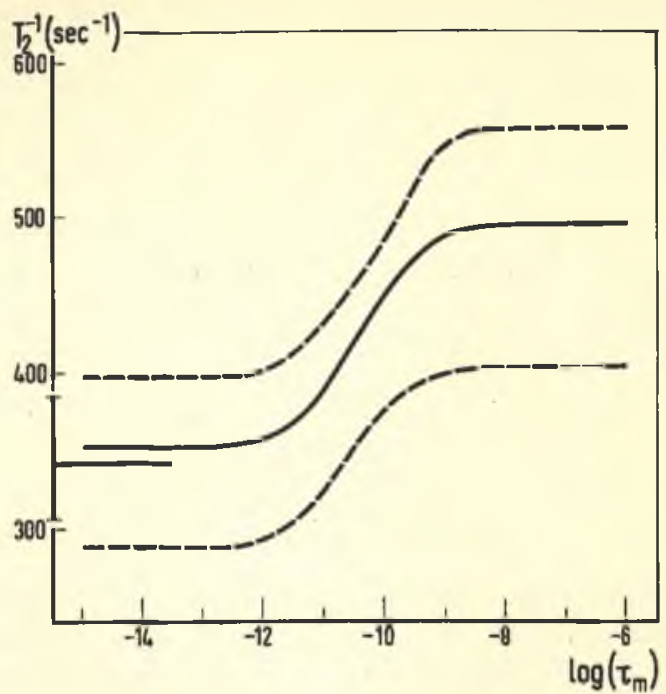

FIG. 7. Theoretical $\tau_{m}$ dependence of $T_{2}^{-1}$ for a $\mathrm{CH}_{3}$ proton in the anion of dmb (solid curve). The dashed curves show the estimated error limits. The horizontal bar at the left-hand side indicates the measured linewidth. The temperature is $30^{\circ} \mathrm{C}$, the concentration of dmb anion $1.9 \mathrm{M}$.

with $T_{2}^{-1}$ predicted for the fast rotational limit. Hence $\tau_{m}$ for the $\mathrm{CH}_{3}$ groups in the dmb anion must lie in the heft horizontal region of the curve in Fig. 7. An upper limit of $\tau_{m}$ is $5 \times 10^{-12} \mathrm{sec}$. This points to a low rotational barrier as might be expected from the geometry of the dmb molecule. The height of this barrier can not be estimated from the temperature dependent measurements because in the fast rotational limit $T_{2}^{-1}$ is independent of the magnitude of $\tau_{m}$. For an estimate of a lower limit for $\tau_{m}$ the free rotor limit in the model of Steele ${ }^{33}$ can be used, which yields $\tau_{m} \geqslant 6 \times 10^{-14} \mathrm{sec}$. In conclusion the $\mathrm{CH}_{3}$ groups in the anion of $\mathrm{dmb}$ rotate too fast to provide an effective relaxation mechanism for the proton spins. We are planning further experiments on compounds containing hindered $\mathrm{CH}_{3}$ groups in order to test the theory in the region where significant contributions to the linewidth due to $\mathrm{CH}_{3}$ rotation are expected.

\section{ACKNOWLEDGMENT}

The authors wish to thank Dr. B. M. P. Hendriks for valuable discussions.

\section{APPENDIX}

If the interaction between the proton spins of the $\mathrm{CH}_{3}$ group is taken into account, the time dependent Hamiltonian $\mathscr{K}_{1}(t)$ is given by

$$
\begin{aligned}
& \mathscr{K}_{1}(t)=\mathscr{K}_{10}+\mathcal{K}_{1 n} \\
& \mathscr{K}_{1 e}=\sum_{j} \mathrm{~S}(t) \cdot \mathrm{A}^{j}(t) \cdot \mathrm{I}_{j}
\end{aligned}
$$




$$
\mathscr{K}_{\mathrm{in}}=\sum_{k, l} \mathrm{I}_{k} \cdot \mathrm{T}^{k l}(t) \cdot \mathrm{I}_{l}
$$

$\mathbf{A}^{j}(t)$ is the total hyperfine interaction tensor for proton $j$ and $\mathrm{T}^{k l}(t)$ is the anisotropic tensor for the dipolar coupling between the $\mathrm{CH}_{3}$ protons $k$ and $l$.

The time derivative of the nuclear magnetization for proton $p, M_{p x}$, is then described as ${ }^{10}$

$$
\begin{aligned}
& \frac{d}{d t} M_{p x}^{*}(t)=-\int_{0}^{\infty} \gamma_{N}\left\{\overline{\left\langle\left[\mathcal{H}_{1 e}^{*}(t-\tau),\left[\mathcal{H C}_{1 e}^{*}(t), I_{p x}\right]\right]\right\rangle}\right. \\
& +\left\langle\overline{\left[\mathcal{H}_{1 n}^{*}(t-\tau),\left[\mathfrak{H}_{1 e}^{*}(t), I_{p x}\right]\right]}\right\rangle \\
& +\left\langle\overline{\left[\mathfrak{F C}_{1 e}^{*}(t-\tau),\left[\mathfrak{H C}_{1 n}^{*}(t), I_{p x}\right]\right]}\right\rangle \\
& \left.\left.+\overline{\left\langle\left[\mathcal{H}_{1 n}^{*}(t-\tau),\left[\mathcal{H}_{1 n}^{*}(t), I_{p x}\right]\right]\right.}\right\rangle\right\} d \tau \text {. }
\end{aligned}
$$

The first term has been considered in our paper, It reduces to $M_{p x} / T_{2 e}$, where $T_{2 e}$ is given by Eq. (7). We will show now that the other terms can be neglected with respect to the first term.

Substituting Eq. (A1) into the second term of Eq. (A2) we get

$$
\begin{aligned}
\sum_{\alpha, \beta, \gamma, \delta} \sum_{j, k_{1} l} \overline{S_{\alpha}^{*}(t-\tau) A_{\alpha \beta}^{j}(t-\tau) T_{\gamma \beta}^{k l}(t)} \\
\times\left\langle\left[I_{j \beta}^{*}(t-\tau),\left[I_{k \gamma}^{*}(t) I_{l 6}^{*}(t), I_{p x}\right]\right]\right\rangle \\
\alpha, \beta, \gamma, \delta=x, y, z \\
j, k, l: \text { running over all protons in } \\
\quad \text { the } \mathrm{CH}_{3} \text { group }
\end{aligned}
$$

The correlation functions in this expression can be written as

$$
\begin{aligned}
\overline{S_{\alpha}^{*}(t-\tau)} \times \overline{A_{\alpha \beta}^{j}(t} & -\tau) T_{\gamma \sigma}^{k l}(t) \\
& =\delta_{\alpha \varepsilon}\left\langle S_{\varepsilon}\right\rangle \overline{A_{\alpha \beta}^{j}(t-\tau) T_{\gamma 6}^{k z}(t)} \\
& \approx \delta_{\alpha \varepsilon} \frac{S(S+1)}{3 k T} \gamma_{e} H_{0} \overline{A_{\alpha \beta}^{j}(t-\tau) T_{\gamma 6}^{k l}(t)} .
\end{aligned}
$$

Since $S(S+1) \gamma_{e} H_{0} / k T \approx 10^{-3}$ in our experiments and $T_{\gamma \theta}^{k l}$ is proportional to $\gamma_{N}^{2}$, whereas $A_{\alpha \beta}^{j}$ is proportional to $\gamma_{\theta} \gamma_{N}$, it can be concluded that this term is negligible (and also, of course, the third one), with respect to the first term (see Sec. II. B).

The contribution of the last term can be estimated from linewidths measured in diamagnetic methyl compounds. An upperlimit is $10 \mathrm{sec}^{-1}$, whereas the methyl proton linewidths in $\mathrm{dmb}^{-}$are larger than $350 \mathrm{sec}^{-1}$.
'G. Herzberg, Infrared and Raman Spectra (Van Nostrand, Princeton, NJ, 1945).

${ }^{2}$ C. C. Lin and J. D. Swalen, Rev. Mod. Phys. 31, 841 (1959).

${ }^{3}$ E. B. Wilson, Jr., Chem. Rev. 27, 17 (1940); C. A. Wulff, J. Chem. Phys. 39, 1227 (1963).

${ }^{4}$ J. J. Rush, T. I. Taylor, and W. W. Havens, J. Chem. Phys. 37, 234 (1962).

5J. H. Freed, J. Chem. Phys. 43, 1710 (1965).

${ }^{6} \mathrm{~S}$. Clough, M. Starr, and N. D. McMillan, Phys. Rev. Lett. 25, 839 (1970); R. B. Davidson and I. Miyagawa, J. Chem. Phys. 52, 1727 (1970).

${ }^{7}$ G. N. La Mar and G. R. Van Hecke, J. Chem. Phys. 52, 5676 (1970).

${ }^{8}$ N. Bloembergen, J. Chem. Phys. 27, 572 (1957).

${ }^{9}$ I. Solomon, Phys. Rev. 99, 559 (1955).

${ }^{10}$ A. Abragam, The Principles of Nuclear Magnetism (Clarendon, Oxford, England, 1961), Chap. VIII.

${ }^{11}$ J. A. M. Van Broekhoven, B. M. P. Hendriks, and E. De Boer, J. Chem. Phys. 54, 1988 (1971).

${ }^{12}$ F. Perrin, J. Phys. Rad. 5, 497 (1934); J. Phys. Rad 7, 1 (1936).

${ }^{13}$ H. Goldstein, Classical Mechanics (Addison-Wesley, Reading, MA, 1959), pp. 107-109.

${ }^{15}$ D. E. Woessner, J. Chem. Phys. 37, 647 (1962).

15 N. Bloembergen, Phys. Rev. 104, 1542 (1956).

${ }^{16} \mathrm{~J}$. H. Freed and G. K. Fraenkel, J. Am. Chem. Soc. 86, 3477 (1964).

${ }^{17}$ D. E. Paul, D. Lipkin, and S. I. Weissman, J. Am. Chem. Soc. 78, 116 (1956).

${ }^{18}$ K. T. Gillen and J. H. Noggle, J. Magn. Reson. 3, 240 (1970).

${ }^{19}$ O. Haworth and R. E. Richards, Progress in Nuclear Magnetic Resonance Spectroscopy (Pergamon, Oxford, England, 1966), Vol. I, Chap. 1.

${ }^{20} \mathrm{G}$. W. Canters, E. de Boer, and H. van Willigen, Chem. Phys. Lett. 1, 627 (1968).

${ }^{21}$ G. W. Canters, E. de Boer, B. M. P. Hendriks, and A. A. K. Klaassen, Proc. Colloq. AMPERE 15, 241 (1969).

${ }^{22}$ K. Ishizu, Bull. Chem. Soc. Jap. 37, 1093 (1964).

${ }^{23}$ C. L. Dodson and A. L. Reddock, J. Chem. Phys. 48, 3226 (1968).

${ }^{24}$ H. M. McConnell, J. Chem. Phys. 24, 764 (1956); S. I. Weissman, J. Chem. Phys. 25, 890 (1956); S. I. Weissman, T. R. Tuttle Jr., and E. de Boer, J. Phys. Chem. 61, 28 (1957).

${ }^{25}$ R. W. Fessenden and R. H. Schuler, J. Chem. Phys. 39, 2147 (1963).

${ }^{26}$ H. M. McConnell and J. Strathdee, Mol. Phys. 2, 129 (1959).

${ }^{27}$ G. W. Canters, Ph.D. thesis, Nijmegen, the Netherlands, 1969.

${ }^{28}$ W. Derbyshire, Mol. Phys. 5, 225 (1962).

${ }^{29}$ E. Clementi and D. L. Raimond, J. Chem. Phys. 38, 2686 (1963).

${ }^{30}$ I. C. Calder, T. Mcl. Spotswood, and C. I. Tanzer, Aust. J. Chem. 20, 1195 (1967).

${ }^{31}$ B. M. P. Hendriks, G. W. Canters, C. Corvaja, J. W. M. de Boer, and E. de Boer, Mol. Phys. 20, 193 (1971).

${ }^{32}$ G. W. Canters and E. de Boer, Mol. Phys. 13, 395 (1967).

${ }^{33}$ W. A. Steele, J. Chem. Phys. 38, 2404 (1963); J. Chem. Phys. 38, 2411 (1963). 
